\title{
INTERPOLATION BETWEEN SOBOLEV AND BETWEEN LIPSCHITZ SPACES OF ANALYTIC FUNCTIONS ON STARSHAPED DOMAINS
}

\author{
EMIL J. STRAUBE
}

\begin{abstract}
We show that on a starshaped domain $\Omega$ in $\mathbf{C}^{n}$ (actually on a somewhat larger, biholomorphically invariant class) the $\mathscr{L}^{p}$-Sobolev spaces of analytic functions form an interpolation scale for both the real and complex methods, for each $p, 0<p \leq \infty$. The case $p=\infty$ gives the Lipschitz scale; here the functor $(,)^{[\theta]}$ has to be considered (rather than $\left.(,)_{[\theta]}\right)$.
\end{abstract}

\section{INTRODUCTION AND RESULTS}

The intermediate spaces between $\mathscr{L}^{p}$-Sobolev and Lipschitz spaces of analytic functions obtained from the complex method have been identified recently in a series of papers by Ligocka [ $\mathrm{Li} \mathrm{1-2]}$ in the case where $\Omega$ is a smoothly bounded strictly pseudoconvex domain in $\mathbf{C}^{n}$ and $1<p$. Certain weighted spaces were considered in [Be]. Both [ $\mathrm{Li} \mathrm{1-2]}$ and [Be] rely heavily on (strict) pseudoconvexity and smoothness of the boundary via estimates for the Bergman projection [Li 1-2] and Henkin-Ramirez type kernels [Be].

It is the purpose of this paper to initiate a study of such interpolation problems in situations where these tools are not available. We do this by studying the case of starshaped $\Omega$ with Lipschitz boundary (actually a somewhat larger, biholomorphically invariant class of domains; compare Remark 1, this section), and $p$ fixed (but $0<p \leq \infty$ ). We show that the $\mathscr{L}^{p}$-Sobolev and the Lipschitz spaces (this is actually the case $p=\infty$; see below) form an interpolation scale for the complex and the real methods. See Theorems 1 and 2 for the exact statements. On the basis of Ligocka's results in the smooth, strictly pseudoconvex case, one may expect the $\mathscr{L}^{p}$-Sobolev spaces of analytic functions to form a double interpolation scale (i.e., interpolation also between different $p$ 's) for the complex method, but the present methods do not seem to give this. The problem is that while in starshaped domains passage between different numbers of derivatives is fairly easy (Lemma $8, \S 2$ ), passage between different $p$ 's is not (fractional powers are multiple valued; regularity of the Bergman projection is not available). Concerning the real method, we note that in a scale of $\mathscr{L}^{p}$-type

Received by the editors March 21, 1988 and, in revised form, May 16, 1988.

1980 Mathematics Subject Classification (1985 Revision). Primary 46E15, 46E35, 32A99, 32A07.

Key words and phrases. Complex interpolation, real interpolation, Sobolev spaces of analytic functions, Lipschitz spaces of analytic functions, starshaped domains. 
spaces (such as the $A^{s, p}$ ), the value of the second interpolation parameter (the " $q$ ") is dictated by the intermediate space. What makes things work here for $p$ fixed is the fact that one is essentially interpolating between the domain of the $k$ th power of an operator and the full space (see $\S 4$ for details). Finally, we point out that the tools we use are elementary: we exploit the fact that membership in the spaces in question is determined by the behavior of radial derivatives only, together with the natural semigroup structure induced by $z \mapsto e^{-t} z$.

Before we can state our results, we must introduce some notation. Let $\Omega$ be a bounded domain in $\mathbf{C}^{n} . \Omega$ is called starshaped with respect to a point $P \in \Omega$ if any ray with origin $P$ has a unique common point with $b \Omega$. In what follows, we assume $P=0$. If we introduce polar coordinates $(r, \theta)$, then $\partial \Omega$ is given by $r=r(\theta)$. We call $\Omega$ starshaped with Lipschitz boundary if $r(\theta)$ satisfies a Lipschitz condition (as a function on the unit sphere $S^{2 n-1}$ ). It is easy to see that this is equivalent to $\Omega$ being starshaped with respect to all points $P$ in a ball centered at $0[\mathrm{M}, \S 1.1 .8]$. In particular, all convex domains are starshaped with Lipschitz boundary.

$O(\Omega)$ denotes the space of analytic functions on $\Omega$ (usually with locally uniform convergence). Let $0<p<\infty, s \in \mathbf{R}$. Denote by $k(s)$ the smallest nonnegative integer greater than or equal to $s$. We denote by $A^{s, p}(\Omega)$ (or simply $\left.A^{s, p}\right)$ the following space:

$$
\begin{aligned}
A^{s, p}(\Omega):=\left\{f \in O(\Omega) /\|f\|_{s, p}^{p}\right. & \\
& \left.:=\int_{\Omega}\left|\delta^{k(s)-s} \nabla^{k(s)} f\right|^{p}+\sum_{j=0}^{k(s)-1}\left|\nabla^{j} f(0)\right|<\infty\right\} .
\end{aligned}
$$

Here, $\delta$ denotes the distance to $b \Omega$, and $\nabla^{j} f$ denotes the vector of all derivatives of order $j$. Then $\|f\|_{s, p}$ is a norm if $1 \leq p$ and a quasi-norm if $0<p<1$. Some comments are in order. First, for $s \in \mathbf{N} k(s)=s$, and it follows easily from Lemma 1 below that $A^{s, p}$ is the usual Sobolev space of analytic functions whose derivatives through order $s$ are $p$ th power integrable. Second, if $s$ is a negative integer, $k(s)=0$, and Lemmas 1 and 8 below imply that the norm in $A^{s, p}(\Omega)$ is equivalent to the usual Sobolev norm for negative integer index. Thus, for $s$ integer, the spaces $A^{s, p}(\Omega)$ are the usual Sobolev spaces of analytic functions. For $s$ fractional, (1) gives a convenient intrinsic description of the intermediate spaces that arise from interpolation.

For $p=\infty$, we set

$$
\begin{aligned}
A^{s, \infty}(\Omega):=\left\{f \in O(\Omega) /\|f\|_{s, \infty}\right. & \\
& \left.:=\sup _{z \in \Omega}\left|\delta^{k(s)+1-s} \nabla^{k(s)+1} f\right|+\sum_{j=0}^{k(s)}\left|\nabla^{j} f(0)\right|<\infty\right\} .
\end{aligned}
$$


For $s>0$, denote by $\Lambda_{s}(\Omega)$ the usual Lipschitz spaces on $\Omega$ [K2, $\left.\S 8.8\right]$. Then $A^{s, \infty}(\Omega)=\Lambda_{s}(\Omega) \cap O(\Omega)$, and $\|f\|_{s, \infty}$ is equivalent to the Lipschitz-s norm; i.e., $A^{s, \infty}(\Omega)$ is (for $s>0$ ) the Lipschitz-s space of analytic functions in $\Omega$. This is contained in Lemma 5 below. Note that $A^{0, \infty}(\Omega)$ is the Bloch space on $\Omega$, not the space of bounded analytic functions. It is in fact for the case of $s \in N$ that we need $k(s)+1$ instead of $k(s)$ in (2). This gives an asymmetry in comparison to (1). This, however, is only superficial: Lemmas 1 and 2 show that one can choose in (1) and (2) any integer $k>k(s)$ as well and obtain an equivalent norm. Thus the Lipschitz scale is truly the $p=\infty$ version of the Sobolev scale (in the case of analytic functions).

Finally, we denote by $\left(A^{s_{0}, p}, A^{s_{1}, p}\right)_{\theta, p},\left(A^{s_{0}, p}, A^{s_{1}, p}\right)_{[\theta]}$, and $\left(A^{s_{0}, p}, A^{s_{1}, p}\right)^{[\theta]}$ the intermediate spaces obtained by the real $(,)_{\theta, p}$ functor and the complex $(,)_{[\theta]}$ and $(,)^{[\theta]}$ functors, respectively. For notation and definitions we refer the reader to [B-L]. Although $\left(A_{0}, A_{1}\right)_{[\theta]}$ and $\left(A_{0}, A_{1}\right)^{[\theta]}$ are not defined for general pairs of quasi-Banach spaces, there is no problem here: the construction is meaningful also in the case $0<p<1$ (and leads to interpolation theorems, since the relevant quantities are subharmonic).

We can now state our main results.

Theorem 1. Let $\Omega$ be starshaped with Lipschitz boundary, let $-\infty<s_{0}, s_{1}<$ $\infty, 0<p<\infty$, and $0<\theta<1$. Then

$$
\left(A^{s_{0}, p}, A^{s_{1}, p}\right)_{\theta, p}=\left(A^{s_{0}, p}, A^{s_{1}, p}\right)_{[\theta]}=\left(A^{s_{0}, p}, A^{s_{1}, p}\right)^{[\theta]}=A^{s, p},
$$

with equivalent norms, where $s=(1-\theta) s_{0}+\theta s_{1}$.

Theorem 2. Let $\Omega$ as above, $-\infty<s_{0}, s_{1}<\infty$, and $0<\theta<1$. Then

$$
\left(A^{s_{0}, \infty}, A^{s_{1}, \infty}\right)_{\theta, \infty}=\left(A^{s_{0}, \infty}, A^{s_{1}, \infty}\right)^{[\theta]}=A^{s, \infty},
$$

with equivalent norms, where $s=(1-\theta) s_{0}+\theta s_{1}$.

Remark 1. The conclusions of Theorems 1 and 2 automatically hold for domains which are biholomorphic images (under biholomorphisms smooth up to the boundary) of starshaped domains (since then the $A^{s, p}$ are isomorphic). Such domains need not be starshaped. It turns out that somewhat stronger versions of Theorems 1 and 2 hold, where the assumptions are invariant under biholomorphisms which are smooth up to the boundary: assume there is a vector field $X$ of type $(1,0)$ with coefficients analytic in $\Omega$ and smooth on $\bar{\Omega}$, such that $X+\bar{X}$ points inside $\Omega$ everywhere on $b \Omega$ (assume here that $b \Omega$ is $C^{1}$ for simplicity). Then the proofs will go through; the contractions $z \mapsto e^{-t} z$ have to be replaced by $z \mapsto \exp (-t(X+\bar{X}))(z)$. In particular, Theorems 1 and 2 remain true for the domains considered in [Ba]. Note that in the starshaped case, $X=\sum_{j=1}^{n} z_{j} \partial / \partial z_{j}$.

On the other hand, the case where these conditions are readily verified is that of a starshaped domain, or of a biholomorphic image of a starshaped domain, and so we have stated our theorems for this case. 
Remark 2. Theorems 1 and 2 also hold for the corresponding spaces of harmonic functions (here, $\Omega \subset \mathbf{R}^{n}$ ); the proofs go through verbatim. For $0<p<$ 1 , one has to use that $|u|^{p}$, although no longer subharmonic, still satisfies a subaveraging inequality with a suitable constant on the right-hand side; see [G, Lemma 3.7, Chapter III] and [F-S, Lemma 2, p. 172].

Remark 3. In Theorem 2 let $s_{0}<s_{1}$. Then $\left(A^{s_{0}, \infty}, A^{s_{1}, \infty}\right)^{[\theta]}=A^{s, \infty} \supsetneqq$ $\left(A^{s_{0}, \infty}, A^{s_{1}, \infty}\right)_{[\theta]}$ in general, since $A^{s_{1}, \infty}$ is dense in $\left(A^{s_{0}, \infty}, A^{s_{1}, \infty}\right)_{[\theta]}$, but not in $A^{s, \infty}$. In [Li 1-2], $\widetilde{\left(^{,}\right)_{[\theta]}}$ was considered for the $A^{s, \infty}$ scale, where denotes completion with respect to $A^{s_{0}, \infty}$ [K-P-S, Chapter IV, §1.6]. It is not hard to see that in our case also $\left(A^{s_{0}, \infty}, A^{s_{1}, \infty}\right)^{[\theta]}=\left(A^{s_{0}, \infty}, A^{s_{1}, \infty}\right)_{[\theta]}$.

The remainder of the paper is organized as follows. In $\S 2$, we collect some auxiliary results and study a family of operators $R_{\zeta}, \zeta \in \mathbf{C}$. These operators are needed in the proofs, given in $\S 3$, of the results concerning the complex methods. In $\S 4$ we give the proofs concerning the real method. $\S 5$ contains versions of Hardy's inequalities when $0<p<1$. These are needed to obtain crucial properties of the operators $R_{\zeta}$, as well as in the proofs in $\S 4$.

Acknowledgment. The author is indebted to H. P. Boas for numerous discussions concerning Sobolev norms of analytic functions.

\section{AuXiliary RESUlts}

In this section we collect various facts and results needed in connection with Theorems 1 and 2. The first part is concerned with Sobolev and Lipschitz norms of analytic functions.

Lemma 1. Let $\Omega \subset \mathbf{C}^{n}$ be a bounded, starshaped domain with Lipschitz boundary, $0<p<\infty, q>-1$, and $s \in \mathbf{N}$. There is a constant $C$ such that for every analytic function $f$ in $\Omega$ we have

$$
C^{-1} \int_{\Omega} \delta^{q}\left|\delta^{s} \nabla^{s} f\right|^{p} \leq \int_{\Omega} \delta^{q}|f|^{p} \leq C\left[\int_{\Omega} \delta^{q}\left|\delta^{s} \nabla^{s} f\right|^{p}+\sum_{j=0}^{s-1}\left|\nabla^{j} f(0)\right|^{p}\right],
$$

where $\delta=\delta(z)$ denotes the distance to the boundary, and $\nabla^{s}$ denotes the vector of all derivatives of order $s$.

Proof. It is enough to prove (1) when $s=1$ (by induction). The left inequality is a standard consequence of the subaveraging properties of $|\nabla f|^{p}$ (see, e.g., the proof of Lemma 1 in [D]).

To prove the right-hand inequality (for $s=1$ ) one can adapt the proof of Proposition 1 in [D], replacing integration in the $y$ direction by integration in the radial direction. Note that the Lipschitz condition on $b \Omega$ implies that the boundary distance and the distance to the boundary measured along the radial direction compare. An alternative argument is as follows. Write $f$ as an $m$-fold integral in the radial direction of $\nabla^{m} f$, where $m$ is chosen so that $q+m p>0$. To estimate this integral, replace $\left|\nabla^{m} f(z)\right|$ by $\left|\nabla^{m} f(z)\right|^{*}$ (see 
(3) below) if $0<p<1$, to get a monotone function, and then apply Hardy's inequality (see Lemma 1, §5) $m$ times (compare the proof of Lemma 8 for details). Since $q+m p>0$, we can apply Lemma 3 below (with $q+m p$ in place of $q$ ), to get rid of the ${ }^{*}$. Finally, the left-hand inequality in (1) already established allows us to trade $m-1$ powers of $\nabla$ against $p(m-1)$ powers of $\delta$, and the right-hand inequality in (1) follows (for $s=1$ ).

Lemma 2. Let $\Omega$ be as above, $s \in \mathbf{N}$, and $q>0$. There is a constant $C$ such that for every analytic function $f$ in $\Omega$ we have

$$
C^{-1} \sup _{\Omega} \delta^{q}\left|\delta^{s} \nabla^{s} f\right| \leq \sup _{\Omega} \delta^{q}|f| \leq C\left[\sup _{\Omega} \delta^{q}\left|\delta^{s} \nabla^{s} f\right|+\sum_{j=0}^{s-1}\left|\nabla^{j} f(0)\right|\right] .
$$

Proof. The left inequality follows by a standard argument, placing a ball of radius comparable to $\delta(z)$ at $z \in \Omega$. The right inequality follows by repeated integration in the radial direction (since $q>0$ ).

If $g$ is a function on $\Omega$, we set

$$
g^{*}(z):=\sup _{t>0}\left|g\left(e^{-t} z\right)\right| ;
$$

i.e., the ${ }^{*}$ denotes the radial maximal function. We will need (for $0<p<1$ ) continuity of the ${ }^{*}$-operator in (volume!) $p$-norms on analytic functions. Under suitable smoothness assumptions on $b \Omega\left(C^{2}\right.$ would do), this follows easily from subharmonicity of $|f|^{p / 2}$ and the usual estimates for the nontangential maximal function on the boundary of a domain. With only Lipschitz boundaries such estimates are available [Da], but the proofs are indirect arguments and it is not clear how the constants involved depend on the domain. Fortunately, what we need here is much more elementary.

Lemma 3. Let $\Omega$ as above, $0<p<\infty$, and $q \geq 0$. Then there is a constant $C$ such that

$$
\int_{\Omega} \delta^{q}\left(|f|^{*}\right)^{p} \leq C \int_{\Omega} \delta^{q}|f|^{p}
$$

for all analytic functions $f$ in $\Omega$.

Proof. Starting with a Whitney decomposition ([Ste, p. 167]; see also [C-W, Theorem 3.2 in the context of general homogeneous spaces]) one can find a sequence of balls $B\left(z_{j}, r_{j}\right)$ with radius $r_{j}$ and center $z_{j} \in \Omega$ such that (for example) $20 r_{j}<\delta\left(z_{j}\right)<100 r_{j}$, each point of $\Omega$ is contained in at least one of the $B\left(z_{j}, r_{j}\right)$, and no point of $\Omega$ is contained in more than $200^{2 n}$ of the larger balls $B\left(z_{j}, 2 r_{j}\right)$. Denote by $\chi_{j}$ the characteristic function of $B\left(z_{j}, r_{j}\right)$. Then

$$
\int_{\Omega} \delta^{q}\left(|f|^{p}\right)^{*} \leq \sum_{j} \int_{\Omega} \delta^{q}\left(\chi_{j}|f|^{p}\right)^{*}
$$


(by Fatou's lemma). $\chi_{j}|f|^{p}$ is supported on (the closure of) $B\left(z_{j}, r_{j}\right)$. Since $r_{j}$ compares to $\delta\left(z_{j}\right),\left(\chi_{j}|f|^{p}\right)^{*}$ is supported on a set whose measure is dominated by $r_{j}^{2 n}$. Moreover, on that set, $\delta$ is dominated by $r_{j}$ (and so $\delta^{q} \lesssim r_{j}^{q}$, since $q \geq 0)$, and $\left(\chi_{j}|f|^{p}\right)^{*}$ is at most equal to $\sup \left\{|f(z)|^{p} \mid z \in B\left(z_{j}, r_{j}\right)\right\}$. Thus

$$
\begin{aligned}
\int_{\Omega} \delta^{q}\left(\chi_{j}|f|^{p}\right)^{*} & \lesssim r_{j}^{2_{n}+q} \sup \left\{|f(z)|^{p} \mid z \in B\left(z_{j}, r_{j}\right)\right\} \\
& \lesssim r_{j}^{q} \int_{B\left(z_{j}, 2 r_{j}\right)}|f(z)|^{p} \lesssim \int_{B\left(z_{j}, 2 r_{j}\right)} \delta^{q}|f|^{p} .
\end{aligned}
$$

Here we have used that for $z \in B\left(z_{j}, r_{j}\right)$,

$$
|f(z)|^{p} \lesssim r_{j}^{-2 n} \int_{B\left(z, r_{j}\right)}|f|^{p} \leq r_{j}^{-2 n} \int_{B\left(z_{j}, 2 r_{j}\right)}|f|^{p} .
$$

We have also used that on $B\left(z_{j}, 2 r_{j}\right) \delta$ compares to $r_{j}$. (4) now follows from (5) and (6) (since no point of $\Omega$ is contained in more than $200^{2 n}$ of the balls $\left.B\left(z_{j}, 2 r_{j}\right)\right)$.

We denote by $X$ the vector field $\sum_{j=1}^{n} z_{j} \partial / \partial z_{j}$. Note that on analytic functions $X$ is essentially differentiation in the radial direction. Proposition 4 shows that membership in $A^{s, p} \quad(p<\infty)$ is determined by the behavior of the radial derivative. $\Omega$ is still starshaped (with respect to 0 ) with Lipschitz boundary.

Proposition 4. Let $0<p<\infty, q>-1$, and $s \in \mathbf{N}$. Then there are a compact subset $K$ of $\Omega$ and a constant $C$ such that for every analytic function $f$ in $\Omega$ we have

$$
\int_{\Omega} \delta^{q}\left|\nabla^{s} f\right|^{p} \leq C\left(\int_{\Omega} \delta^{q}\left|X^{s} f\right|^{p}+\int_{K}|f|^{p}\right) .
$$

Proof. By induction (note that $X f$ is also analytic), it suffices to consider the case $s=1$. Thus one must show that

$$
\int_{\Omega} \delta^{q}|\nabla f|^{p} \lesssim \int_{\Omega} \delta^{q}|X f|^{p}+\int_{K}|f|^{p} .
$$

This follows by slightly modifying the arguments in [D]. We also sketch a somewhat different argument. We can write $\nabla f$ as an integral in the radial direction of $X \nabla f$. Dominating $|X \nabla f|$ by $|\nabla X f|+|\nabla f|$ (since $[X, \nabla]$ is first order) and applying Hardy's inequality (Lemma $1, \S 5$ ) to the integral in the radial direction, one obtains (see again the proof of Lemma 8 for details)

$$
\int_{\Omega} \delta^{q}|\nabla f|^{p} \lesssim \int_{\Omega} \delta^{q}\left(\delta|\nabla X f|^{*}\right)^{p}+\int_{\Omega} \delta^{q}\left(\delta|\nabla f|^{*}\right)^{p}+\int_{K}|f|^{p} .
$$

If $q+p>0$, we apply Lemma 3 and the left-hand inequality of Lemma 1, and repeat the same kind of argument for $\int_{\Omega} \delta^{q}|f|^{p}$ to obtain (8). In general, we have to choose $m$ so that $q+m p>0$ and then write $\nabla f$ as an iterated $m$-fold integral of $X^{m} \nabla f$. 
Membership in $\Lambda_{s}(\Omega) \cap O(\Omega)$ is equivalent to a growth condition on the derivatives of $f$. This is known, but a proof for the case of Lipschitz boundary seems to be hard to find in the literature; for the case of $C^{2}$-boundary (but harmonic functions) compare, however, [K1, Proposition 4.1].

Lemma 5. Let $0<s<\infty, k$ an integer $>s$. An analytic function $f$ belongs to $\Lambda_{s}(\Omega)$ if and only if

$$
\sup _{z \in \Omega} \delta^{k-s}(z)\left|\nabla^{k} f(z)\right|<\infty
$$

Moreover,

$$
\sup _{z \in \Omega} \delta^{k-s}(z)\left|\nabla^{k} f(z)\right|+\sum_{j=0}^{k-1}\left|\nabla^{j} f(0)\right|
$$

provides a norm which is equivalent to the Lipschitz-s norm on $\Lambda_{s}(\Omega) \cap O(\Omega)$. Proof. We indicate how to adapt the proof of Zygmund's original result (essentially Lemma 5 for the unit disk) given in Duren's book [Du, proof of Theorem 5.3]. The fact that $f \in \Lambda_{s}(\Omega)$ implies (10) follows by placing a ball of radius $\delta(z) / 2$ at $z \in \Omega$ and then using the symmetries of the Poisson kernel for this ball; otherwise the argument is as in [Du]. For the reverse direction, the argument is almost the same as in [Du]; again the Lipschitz condition on $b \Omega$ implies that the (true) boundary distance and the one measured along the radial direction compare. The fact that our norms are over the interior is no essential complication.

We next show that in (10) and (11) it suffices to consider radial derivatives (this is the analog of Proposition 1.4). For the case of $C^{2}$ boundary, compare again [K1, Theorem 7.5].

Lemma 6. Let $\Omega$ be as above, $k \in \mathbf{N}$, and $q>0$. There exist a constant $C$ and a compact subset $K$ of $\Omega$ such that for all analytic functions $f$ in $\Omega$

$$
\sup _{z \in \Omega}\left\{\delta(z)^{q}\left|\nabla^{k} f(z)\right|\right\} \leq C\left(\sup _{z \in \Omega} \delta(z)^{q}\left|X^{k} f(z)\right|+\sup _{z \in K}|f(z)|\right) .
$$

Proof. The proof is by induction on $k$. First, let $k=1$. Let $K$ be any compact subset of $\Omega$ that contains a neighborhood of 0 . Set $M:=\sup _{z \in \Omega}\left\{\delta^{q}|X f|\right\}+$ $\sup _{z \in K}\{|f|\}$. Then $|X f(z)| \leq M / \delta(z)^{q}$, and so (since $\left.\delta(r \theta) \approx r(\theta)-r\right)$

$$
|f(z)| \lesssim \begin{cases}M\left(\frac{1}{\delta(z)^{q-1}}+1\right), & q \neq 1, \\ M(|\log \delta(z)|+1), & q=1 .\end{cases}
$$

Therefore, in either case of (13),

$$
|\nabla f(x)| \lesssim \frac{M}{\delta(z)^{q+1}}
$$


Also,

$$
|X \nabla f(z)| \lesssim|\nabla X f(z)|+|\nabla f(z)| \lesssim M / \delta(z)^{q+1}+M / \delta(z)^{q+1},
$$

since $X f$ is analytic (for the first term) and in view of (14) (for the second term). (15) gives the desired conclusion upon integration in the radial direction (note that $X=r \partial / \partial r$ on analytic functions).

Next, let $k>1$ and assume the lemma holds for all integers up to $k-1$. By the induction hypothesis (note again that $X^{k-1} f$ is analytic)

$$
\begin{aligned}
\sup _{z \in \Omega}\left\{\delta^{q}\left|\nabla^{k} f\right|\right\} \lesssim & \sup _{z \in \Omega}\left\{\delta^{q}\left|X^{k-1} \nabla f\right|\right\}+\sup _{z \in K}|\nabla f| \\
\lesssim & \sup _{z \in \Omega}\left\{\delta^{q}\left|\nabla X^{k-1} f\right|\right\}+\sup _{z \in \Omega}\left\{\delta^{q}\left|\nabla^{k-1} f\right|\right\}+\sup _{z \in K}|\nabla f| \\
\lesssim & \sup _{z \in \Omega}\left\{\delta^{q}\left|X^{k} f\right|\right\}+\sup _{z \in K^{\prime}}\left|X^{k-1} f\right| \\
& +\sup _{z \in \Omega}\left\{\delta^{q}\left|X^{k-1} f\right|\right\}+\sup _{z \in K^{\prime \prime}}|f|+\sup _{z \in K}|\nabla f| \\
\lesssim & \sup _{z \in \Omega}\left\{\delta^{q}\left|X^{k} f\right|\right\}+\sup _{z \in K^{\prime \prime \prime}}|f|,
\end{aligned}
$$

where $K^{\prime \prime \prime}$ is sufficiently large. In the last inequality in (16), we have again used integration in the radial direction to estimate $X^{k-1} f$ from the bound on $X^{k} f$ (similar to (13)).

Remark 1. Lemmas 5 and 6 together show in particular that if an analytic function is in $\Lambda_{s}$ for all 1-dimensional complex slices through 0 , with $\Lambda_{s}$-norm bounded independently of the slice, then $f$ is in $\Lambda_{s}$ on $\Omega$.

On $O(\Omega)$, we now define a one-parameter family of operators; here, the parameter $\zeta$ is in $\mathbf{C}$. The operator will depend analytically on $\zeta$. For $\zeta$ given, choose a nonnegative integer $m$ such that $\operatorname{Re} \zeta+m>0$. Then we set

$$
R_{\zeta} f(z):=\frac{(-1)^{m}}{\Gamma(\zeta+m)} \int_{0}^{\infty} t^{\zeta+m-1} \frac{d^{m}}{d t^{m}}\left(e^{-t} f\left(e^{-t} z\right)\right) d t .
$$

Here $\Gamma(\cdot)$ denotes the gamma function. Integration by parts shows that the right-hand side of (17) is independent of the choice of $m$; also note that since $\operatorname{Re} \zeta+m-1>-1$, the integral in (17) is absolutely convergent. For $f \in O(\Omega)$, fixed, $R_{\zeta} f$ depends analytically on $\zeta$ (in the topology of locally uniform convergence). For a general $\zeta$, we find after performing the $m$-fold differentiation with respect to $t$,

$$
R_{\zeta} f(z)=\frac{1}{\Gamma(\zeta+m)} \int_{0}^{\infty} t^{\zeta+m-1} e^{-t}\left(\sum_{j=0}^{m}\left(\begin{array}{c}
m \\
j
\end{array}\right) X^{j} f\left(e^{-t} z\right)\right) d t
$$

Note that if $\tau_{t}$ is the semigroup on $A^{0, p}$ defined by $\tau_{t} f(z):=f\left(e^{-t} z\right)$, then its generator is $-X$, and $R_{\zeta}$ is essentially the $\zeta$ th power of $(1+X)^{-1}$. (See [H$\mathrm{Ph}$ ] for the functional calculus for the resolvent of a semigroup, via the Laplace transform.) 
Lemma 7. $R_{0}$ is the identity, i.e., $R_{0} f=f$, and

$$
R_{\zeta_{1}} R_{\zeta_{2}}=R_{\zeta_{2}} R_{\zeta_{1}}=R_{\zeta_{1}+\zeta_{2}}
$$

Proof. That $R_{0} f=f$ is immediate from (17); choose $m=1$. To prove (19), first let $\operatorname{Re} \zeta_{1}>0, \operatorname{Re} \zeta_{2}>0$ (in order to simplify (17)). A direct computation, using the fact that

$$
\int_{0}^{1}(1-\sigma)^{\zeta_{1}-1} \sigma^{\zeta_{2}-1} d \sigma=\frac{\Gamma\left(\zeta_{1}\right) \Gamma\left(\zeta_{2}\right)}{\Gamma\left(\zeta_{1}+\zeta_{2}\right)}
$$

then shows that

$$
R_{\zeta_{1}} R_{\zeta_{2}} f(z)=R_{\zeta_{2}} R_{\zeta_{1}} f(z)=R_{\zeta_{1}+\zeta_{2}} f(z) .
$$

Keeping $f$ and $z \in \Omega$ fixed, (20) extends to all $\zeta_{1}, \zeta_{2}$ by analytic continuation.

Remark 2. Note in particular that $R_{\zeta}$ and $R_{-\zeta}$ are inverses of each other.

Remark 3. Note further that $R_{-1}=1+X$, hence $R_{-k}=(1+X)^{k}$. This is immediate from (17), but it should of course be viewed in the context of the remarks made before the statement of Lemma 7.

Lemma 8. Let $0<p \leq \infty, s \in \mathbf{R}, \zeta \in C$. Then $R_{\zeta}$ is an isomorphism from $A^{s, p}(\Omega)$ onto $A^{s+\operatorname{Re} \zeta, p}(\Omega)$. Moreover, if $a \leq \operatorname{Re} \zeta \leq b$, there are constants $C_{1}=$ $C_{1}(a, b, s, p)$ and $C_{2}=C_{2}(a, b)$ such that $\left\|R_{\zeta} f\right\|_{s+\operatorname{Re} \zeta, p} \leq C_{1} e^{C_{2}|\operatorname{Im} \zeta|}\|f\|_{s, p}$. Proof. It suffices to show continuity of $R_{\zeta}$ (all $\zeta$ ). The fact that $R_{\zeta}$ is then actually an isomorphism follows from Lemma 7 . The proof will give the required control on the constants. We first do the case $0<p<\infty$. Choose an integer $k>\max \{0, s, s+\operatorname{Re} \zeta\}$. Then, by Lemma 1 and Proposition 4 .

$$
\left\|R_{\zeta} f\right\|_{s+\operatorname{Re} \zeta, p}^{p} \lesssim \int_{\Omega}\left(\delta^{k-s-\operatorname{Re} \zeta}\left|X^{k} R_{\zeta} f\right|\right)^{p}+\sum_{j=0}^{k-1}\left|\nabla^{j} R_{\zeta} f(0)\right|^{p}+\int_{K}\left|R^{\zeta} f\right|^{p} .
$$

It is implicit in the proofs of Lemma 1 and Proposition 4 that the constant in (21) can be chosen independently of $\zeta$, as long as $a \leq \operatorname{Re} \zeta \leq b$. The last two contributions in (21) can be estimated by $\sup _{K}\left|R^{\zeta} f\right|$ for a suitably large compact subset $K$ of $\Omega$, and hence by the sup-norm of derivatives of $f$ over a (possibly larger) compact subset of $\Omega$. These in turn are estimated by $\|f\|_{s, p}$. It remains to estimate the integral in (21).

To this end, choose a nonnegative integer $m$ such that $a+m>1$. Then

$$
X^{k} R_{\zeta} f(z)=\frac{(-1)^{k}}{\Gamma(\zeta+m)} \int_{0}^{\infty} t^{\zeta+m-1} e^{-t}\left(\sum_{j=0}^{m}\left(\begin{array}{c}
m \\
j
\end{array}\right) X^{j+k} f\left(e^{-t} z\right)\right) d t
$$

We consider the term in (22) corresponding to $j=m$; the other terms can be treated similarly (and in fact behave even better). Fix $z_{0} \in b \Omega$, and let $z=e^{-x} z_{0}$, and set

$$
h(x):=\int_{0}^{\infty} t^{\zeta+m-1} e^{-t} X^{m+k} f\left(e^{-(t+x)} z_{0}\right) d t .
$$


Then (note that $\operatorname{Re} \zeta+m-1>0$ )

$$
\begin{aligned}
\left|e^{-x} h(x)\right| & \leq \int_{0}^{\infty} t^{\operatorname{Re} \zeta+m-1} e^{-(t+x)}\left|X^{m+k} f\left(e^{-(t+x)} z_{0}\right)\right| d t \\
& \leq \int_{0}^{\infty}(t+x)^{\operatorname{Re} \zeta+m-1} e^{-(t+x)}\left|X^{m+k} f\left(e^{-(t+x)} z_{0}\right)\right| d t \\
& =\int_{x}^{\infty} \sigma^{\operatorname{Re} \zeta+m-1} e^{-\sigma} \mid X^{m+k} f\left(e^{-\sigma} z_{0}\right) d \sigma \\
& \leq \int_{x}^{\infty} \sigma^{\operatorname{Re} \zeta+m-1} e^{-\sigma}\left|X^{m+k} f\right|^{*}\left(e^{-\sigma} z_{0}\right) d \sigma
\end{aligned}
$$

where the ${ }^{*}$ is to be interpreted as a null symbol when $p \geq 1$ (i.e., $\left|X^{m+k} f\right|^{*}=$ $\left|X^{m+k} f\right|$ in this case) and as denoting the maximum of $\left|X^{m+k} f\right|$ on the line segment joining 0 and $z=e^{-\sigma} z_{0}$ otherwise. The point is that $\left|X^{m+k} f\right|^{*}$ and hence $e^{-\sigma}\left|X^{m+k} f\left(e^{-\sigma} z_{0}\right)\right|^{*}$ are nonincreasing. This allows us to apply Hardy's inequality (see Lemma $1, \S 5$ ) even for $0<p<1$. The result is

$$
\begin{aligned}
\int_{0}^{\infty} & \left(x^{k-s-\operatorname{Re} \zeta} e^{-x}|h(x)|\right)^{p} d x \\
& \lesssim \int_{0}^{\infty}\left(x^{k-s-\operatorname{Re} \zeta} x^{\operatorname{Re} \zeta+m} e^{-x}\left|X^{m+k} f\right|^{*}\left(e^{-x} z_{0}\right)\right)^{p} d x \\
& =\int_{0}^{\infty}\left(x^{k+m-s} e^{-x}\left|X^{m+k} f\right|^{*}\left(e^{-x} z_{0}\right)\right)^{p} d x \\
& \lesssim \int_{0}^{1}\left(x^{k+m-s}\left|X^{m+k} f\right|^{*}\left(e^{-x} z_{0}\right)\right)^{p} d x+\|f\|_{s, p}^{p} .
\end{aligned}
$$

In the last inequality we have used to fact that for $x \geq 1, f\left(e^{-x} z_{0}\right)$ is dominated independently of $x$ and $z_{0} \in b \Omega$ by $\|f\|_{s, p}$, since then $e^{-x} z_{0}$ is contained in a fixed compact subset of $\Omega$.

As mentioned above, the terms in (22) arising for $0 \leq j \leq m-1$ can be treated similarly, and the resulting estimate for $X^{k} R_{\zeta} f$ becomes

$$
\begin{array}{r}
\int_{0}^{1}\left(x^{k-s-\operatorname{Re} \zeta}\left|X^{k} R_{\zeta} f\left(e^{-x} z_{0}\right)\right|\right)^{p} d x \lesssim \frac{1}{\Gamma(\zeta+m)^{p}} \int_{0}^{\infty}\left(x^{k-s-\operatorname{Re} \zeta} e^{-x}|h(x)|\right)^{p} d x \\
\lesssim \frac{1}{\Gamma(\zeta+m)^{p}}\left[\int_{0}^{1}\left(x^{k+m-s}\left|X^{m+k} f\right|^{*}\left(e^{-x} z_{0}\right)\right)^{p} d x+\|f\|_{s, p}^{p}\right] .
\end{array}
$$

Noting that to estimate the integral in (21), it suffices to estimate the integral over $\Omega \backslash K$, for an arbitrary compact subset $K$, and that $\delta(z)$ compares to $r(\theta)-r \quad(z=r \theta)$, we get from (26) (by splitting the integration over slices and radial direction)

$$
\begin{aligned}
\int_{\Omega}\left(\delta^{k-s-\operatorname{Re} \zeta}\left|X^{k} R_{\zeta} f\right|\right)^{p} & \lesssim \frac{1}{\Gamma(\zeta+m)^{p}}\left[\int_{\Omega}\left(\delta^{k+m-s}\left|X^{k+m} f\right|^{*}\right)^{p}+\|f\|_{s, p}^{p}\right] \\
& \lesssim \frac{1}{\Gamma(\zeta+m)^{p}}\left[\int_{\Omega}\left(\delta^{k+m-s}\left|X^{k+m} f\right|\right)^{p}+\|f\|_{s, p}^{p}\right] .
\end{aligned}
$$


The last inequality in (27) (nontrivial only for $0<p<1$ ) follows from Lemma 3. (27) proves Lemma 8 (for $p<\infty$ ), except for the behavior of $1 / \Gamma(\zeta+m)$. But Stirling's formula (for example [H, Theorems $8.5 \mathrm{~b}$ and $8.5 \mathrm{a}]$ ) shows that $1 / \Gamma(\zeta+m) \leq C_{1} e^{C_{2}|\operatorname{Im} \zeta|}$ with $C_{1}$ and $C_{2}$ as required.

The case $p=\infty$ can be done using Lemmas 2,5 , and 6 . Since only sup-norms have to be estimated, the situation is considerably simpler: it suffices in (24) to estimate $\left|X^{m+k} f\left(e^{-\sigma} z_{0}\right)\right|$ by $\|f\|_{s, p} / \sigma^{k+m-s}$ to get the required estimate, i.e., $\left|e^{-x} h(x)\right| \lesssim\|f\|_{s, p} / x^{k-s-\operatorname{Re} \zeta}$. We leave the details to the reader.

This completes the proof of Lemma 8.

We will also need that $R_{\zeta} f$, for $f$ fixed, is analytic in $\zeta$ as a function with values in some of the (quasi) Banach spaces that we are considering. This is contained in the next lemma.

Lemma 9. Fix $f \in A^{0, p}(\Omega), 0<p \leq \infty$. Then $R_{\zeta} f$ is an analytic $A^{0, p}(\Omega)$ valued function in $\{\operatorname{Re} \zeta>0\}$. If $p<\infty$, this function is continuous on $\{\operatorname{Re} \zeta \geq 0\}$, with $R_{i s}=R_{1+i s}(f+X f), s \in \mathbf{R}$.

Proof. For analyticity, it suffices to consider $A_{\zeta} f(z):=\int_{0}^{\infty} t^{\zeta-1} e^{-t} f\left(e^{-t} z\right) d t$. Then $(\partial / \partial \zeta)\left(A_{\zeta} f(z)\right)=\int_{0}^{\infty} \log t \cdot t^{\zeta-1} e^{-t} f\left(e^{-t} z\right) d t$ (for $z \in \Omega$ fixed). But the arguments used in the proof of Lemma 8 show that $(\partial / \partial \zeta)\left(A_{\zeta} f(z)\right) \in A^{0, p}(\Omega)$ and that the difference quotients converge not only for $z$ fixed, but in $A^{0, p}(\Omega)$ (use that $\log t \cdot t^{\operatorname{Re} \zeta / 2}$ is bounded near 0 ).

Now let $p<\infty$. Fix $\zeta_{0}$ with $\operatorname{Re} \zeta_{0}=0$, and let $\zeta_{n} \rightarrow \zeta_{0}$ as $n \rightarrow \infty$. Integration by parts shows that $R_{\zeta} f=R_{\zeta+1}(f+X f)$, for $\operatorname{Re} \zeta>0$. Therefore,

$$
\lim _{n \rightarrow \infty} R_{\zeta_{n}} f=R_{\zeta_{0}+1}(f+X f),
$$

provided $f \in A^{1, p}(\Omega)$ (so that $X f \in A^{0, p}(\Omega)$ ), by what we have already shown. But $A^{1, p}$ is dense in $A^{0, p}$ (use dilatations), and the norms of $R_{\zeta_{n}}$ (as operators on $A^{0, p}$ ) are bounded independently of $n$ (Lemma 8). It follows that (28) holds for all $f \in A^{0, p}$ (note that $f \mapsto R_{\zeta_{0}+1}(f+X f)$ is a continuous operator on $A^{0, p}$, according to Lemma 8 ). This completes the proof of Lemma 9.

\section{THE COMPLEX METHOD}

We begin by showing that

$$
\left(A^{s_{0}, p}, A^{s_{1}, p}\right)_{[\theta]}=A^{s, p}, \quad p<\infty,
$$

where

$$
s=(1-\theta) s_{0}+\theta s_{1} .
$$

Without loss of generality, let $s_{0}<s_{1}$. First, let $f \in\left(A^{s_{0}, p}, A^{s_{1}, p}\right)_{[\theta]}$. Fix $\mathscr{E}>$ 0 . Then there is an analytic (that is, the complex derivative exists at each point 
$\zeta$ of $S$ ) function $F(\zeta)$ in the strip $S:=\{\zeta \in \mathbf{C} \mid 0<\operatorname{Re} \zeta<1\}$, with values in $A^{S_{0}, p}$, bounded and continuous on the closure $\bar{S}$ of $S$, with the following additional properties. The value of $F$ at $\theta$ is $f$; i.e., $F(\theta)=f \in A^{S_{0}, p}$. The functions $t \mapsto F(j+i t) \quad(j=0,1)$ are continuous functions from the real line into $A^{s_{j}, p}$, which tend to zero as $|t| \rightarrow \infty$. Finally,

$$
\max \left\{\sup \|F(i t)\|_{s_{0}, p}, \sup \|F(1+i t)\|_{s_{1}, p}\right\} \leq\|f\|_{[\theta]}+\mathscr{E} .
$$

Here, $\|f\|_{[\theta]}$ denotes the norm of $f$ in $\left(A^{s_{0}, p}, A^{s_{1}, p}\right)_{[\theta]}$. Choose a positive integer $k>s_{1}$, and consider

$$
h(\zeta):=\int_{\Omega_{\varepsilon}}\left|\delta^{k-(1-\zeta) s_{0}-\zeta s_{1}}(z) \nabla_{z}^{k} F(\zeta, z)\right|^{p} d V(z),
$$

where $\nabla_{z}$ denotes the gradient with respect to $z, F(\zeta, z)$ is $F(\zeta)$ evaluated at $z \in \Omega$, and $\Omega_{\mathscr{E}}:=\{z \in \Omega \mid \delta(z)>\mathscr{E}\}$. Then $h(\zeta)$ is bounded and continuous on $\bar{S}$ and subharmonic on $S$. Moreover

$$
\begin{aligned}
|h(1+i t)| & =\int_{\Omega_{\mathscr{E}}}\left(\delta(z)^{k-s_{1}}\left|\nabla_{z}^{k} F(1+i t, z)\right|\right)^{p} d V(z) \\
& \lesssim\left(\|F(1+i t)\|_{s_{1}, p}\right)^{p} \lesssim\left(\|f\|_{[\theta]}+\mathscr{E}\right)^{p},
\end{aligned}
$$

by Lemma 1 (with constant independent of $\mathscr{E}$ since $\int_{\Omega_{\varepsilon}} \leq \int_{\Omega}$ ). Similarly,

$$
|h(i t)| \lesssim\left(\|F(i t)\|_{s_{0}, p}\right)^{p} \lesssim\left(\|f\|_{[\theta]}+\mathscr{E}\right)^{p} .
$$

The three-line theorem yields (in view of (4), (5))

$$
|h(\theta)| \lesssim\left(\|f\|_{[\theta]}+\mathscr{E}\right)^{p} .
$$

Since the constant in (6) does not depend on $\mathscr{E}$, letting $\mathscr{E}$ tend to zero yields

$$
\int_{\Omega}\left|\delta(z)^{k-s} \nabla_{z}^{k} F(\zeta, z)\right|^{p} d V(z) \lesssim\left(\|f\|_{[\theta]}\right)^{p},
$$

which is the desired conclusion $\left(\sum_{j=0}^{k-1}\left|\nabla^{j} f(0)\right|^{p}\right.$ is similarly seen to be bounded by $\left.\|f\|_{[\theta]}+\mathscr{E}\right)$. A similar argument occurs in [Be] in the proof of Theorem 1.3.

Next, assume that $f \in A^{s, p}$. Set

$$
F(\zeta, z):=e^{\zeta^{2}-\theta^{2}} R_{\left(s_{1}-s_{0}\right)(\zeta-\theta)} f(z) .
$$

For $\zeta \in S$ fixed, $F(\zeta) \in A^{S+\left(s_{1}-s_{0}\right)(\operatorname{Re} \zeta-\theta), p}=A^{s_{0}+\left(s_{1}-s_{0}\right) \operatorname{Re} \zeta, p}$ by Lemma 8 . Thus $F$ has values in $A^{s_{0}, p}$. Also, as a function with values in $A^{s_{0}, p}, F$ is analytic in $S$; in view of Lemma 8, this follows from Lemma 9. Of course, $F(\theta)=f$. And

$$
\begin{aligned}
\|F(\zeta)\|_{s_{0}+\operatorname{Re} \zeta\left(s_{1}-s_{0}\right), p} & \lesssim\left|e^{\zeta^{2}-\theta^{2}}\right|\left\|R_{\left(s_{1}-s_{0}\right)(\zeta-\theta)} f\right\|_{s_{0}+\operatorname{Re} \zeta\left(s_{1}-s_{0}\right), p} \\
& \lesssim e^{-(\operatorname{Im} \zeta)^{2}} e^{C|\operatorname{Im} \zeta|}\|f\|_{s, p}
\end{aligned}
$$


thus $F$ is bounded on $S$ (as an $A^{S_{0}, p}$-valued function). Lemma 9 (again in view of Lemma 8 ) also shows that $F$ is continuous on $\bar{S}$. (9) further shows that for $j=1,2, F(j+i t) \rightarrow 0$ as $(t) \rightarrow \infty$, and finally that

$$
\max \left\{\sup \|F(i t)\|_{s_{0}, p}, \sup \|F(1+i t)\|_{s_{1}, t}\right\} \lesssim\|f\|_{s, p} .
$$

This completes the proof of (1) above.

Next, note that in general $\left(A_{0}, A_{1}\right)_{[\theta]} \subset\left(A_{0}, A_{1}\right)^{[\theta]}$. The argument in [BL] (proof of Theorem 4.3.1) also works for $0<p<1$ (if instead of the quasi-norm $\|f\|_{s, p}$ one considers the distance to the origin $\left.d(0, f)\right)$. To see that $\left(A^{s_{0}, p}, A^{s_{1}, p}\right)^{[\theta]} \subset A^{s, p}$, choose $G(\zeta)$, analytic in $S$ (values in $A^{s_{0}, p}$ ), continuous on $\bar{S}$, with $\|G(\zeta)\|_{s_{0}, p} \leq C(1+|\zeta|)$, such that

$$
\begin{gathered}
\max \left(\sup _{t_{1}, t_{2}}\left\|\frac{G\left(i t_{1}\right)-G\left(i t_{2}\right)}{t_{1}-t_{2}}\right\|_{A^{s_{0}, p}} \sup _{t_{1}, t_{2}}\left\|\frac{G\left(1+i t_{1}\right)-G\left(1+i t_{2}\right)}{t_{1}-t_{2}}\right\|_{A^{s_{1}, p}}\right) \\
<\left\|G^{\prime}(\theta)\right\|_{\left(A^{s_{0}, p}, A^{s_{1}, p}\right)[\theta]}+\mathscr{E} .
\end{gathered}
$$

For any integer $k>s_{1}$, consider

$$
h(\zeta):=\int_{\Omega_{\varepsilon}}\left|\delta(z)^{k-(1-\zeta) s_{0}-\zeta s_{1}} \nabla_{z}^{k}\left(\frac{G(\zeta, z)-G(\zeta+i t, z)}{t}\right)\right|^{p} d V(z) .
$$

With (11) and the three-line theorem, and using Fatou's lemma to let $t \rightarrow 0$, we find (after also letting $\mathscr{E} \rightarrow 0$ )

$$
\left\|G^{\prime}(\theta)\right\|_{s, p} \leq C\left\|G^{\prime}(\theta)\right\|_{\left(A^{s_{0}, p}, A^{\left.s_{1}, p\right)}[\theta]\right.} .
$$

This completes the proof of the assertions concerning the complex methods in Theorem 1.

We now prove the assertions concerning the complex method in Theorem 2. To show that $\left(A^{S_{0}, \infty}, A^{S_{1}, \infty}\right)^{[\theta]}$ is (continuously) contained in $A^{s, \infty}$, consider the auxiliary function

$$
h_{z}(\zeta):=\left|\delta(z)^{k-(1-\zeta) s_{0}-\zeta s_{1}} \nabla_{z}^{k}\left(\frac{G(\zeta, z)-G(\zeta+i t, z)}{t}\right)\right|
$$

for $z \in \Omega$ fixed. The rest of the argument is as above.

Next, assume $f \in A^{s, \infty}$, and set

$$
F(\zeta, z):=e^{\zeta^{2}-\theta^{2}} R_{\left(s_{1}-s_{0}\right)(\zeta-\theta)} f(z)
$$

and

$$
G(\zeta, z):=\int_{\theta}^{\zeta} F(\sigma, z) d \sigma .
$$

Note that for $z \in \Omega$ fixed, $F(\zeta, z)$ is analytic on $\bar{S}$ (it is in fact entire). In view of Lemma 8 and Lemma $9, F$ as an $A^{s_{0}, \infty}$-valued function is analytic in $S$ and bounded by $\|f\|_{s, \infty}$. Also, $F(1+i t, z)$ as a function of $t$ with values in $A^{s_{1}, \infty}$ is bounded by $\|f\|_{s, \infty}$. From these observations it follows 
that $G$ is in the class of functions considered for the $(,)^{[\theta]}$ functor, and that $f(z)=(\partial G / \partial \zeta)(\theta, z) \in\left(A^{s_{0}, \infty}, A^{s_{1}, \infty}\right)^{[\theta]}$ with its norm in $\left(A^{s_{0}, \infty}, A^{s_{1}, \infty}\right)^{[\theta]}$ dominated by $\|f\|_{s, \infty}$. This completes the proof of Theorem 2 , as far as the complex method is concerned.

\section{THE REAL METHOD}

In this section, we prove the statements in Theorems 1 and 2 concerning the real method. We first turn to Theorem 1. By a standard reiteration argument, we may assume that both $s_{0}$ and $s_{1}$ are integers. In view of Lemma 8, we can assume that $s_{0}=0$ and $s_{1}=k>0(k \in N)$. Thus we must show that

$$
\left(A^{0, p}, A^{k, p}\right)_{\theta, p}=A^{k \theta, p}, \quad 0<p<\infty .
$$

First, assume $f \in\left(A^{0, p}, A^{k, p}\right)_{\theta, p}$. For each $t$, there exist $f_{0}(t) \in A^{0, p}, f_{1}(t) \in$ $A^{k, p}$, such that $f=f_{0}(t)+f_{1}(t)$ and such that

$$
\left\|f_{0}(t)\right\|_{0, p}+t^{k}\left\|f_{1}(t)\right\|_{k, p} \leq 2 K\left(t^{k}, f\right) .
$$

(Here, $K(t, f)$ is the standard $K$-functional used for the real interpolation method.) Let $f \in C^{\infty}(\bar{\Omega}) \cap O(\Omega)$. We will denote by "c.s." terms that are supported in a compact subset of $\Omega$. Such terms are estimated by any one of the norms involved (by standard arguments). Then

$$
\left(\|f\|_{k \theta, p}\right)^{p}=\int_{\Omega}\left(\delta^{k-\theta k}\left|\nabla^{k} f\right|\right)^{p}+\text { c.s. } \lesssim \int_{0}^{1} \int_{b \Omega_{t}} \delta^{(k-\theta k) p}\left|\nabla^{k} f\right|^{p} d t d \sigma_{t}+\text { c.s. }
$$

Here, $d \sigma_{t}$ is surface measure on $\Omega_{t}:=e^{-t} \Omega$ (note that $b \Omega_{t}$ is Lipschitz). The last quantity in $(3)$ is dominated by (note that $\delta(z) \approx t$ )

$$
\int_{0}^{1} t^{k p-\theta k p} \int_{b \Omega_{t}}\left|\nabla^{k} f\right|^{p} d \sigma_{t} d t+\text { c.s. } \lesssim \int_{0}^{1} t^{k p-\theta k p}\left(\int_{\Omega_{t}}\left|\nabla^{k} f\right|^{p} d V(z)\right) \frac{d t}{t}+\text { c.s. }
$$

by integration by parts (note that we are not claiming equality; the relevant density terms are bounded from above and below)

$$
\begin{aligned}
& \lesssim \int_{0}^{1} t^{k p-\theta k p} \int_{\Omega_{t}}\left(\left|\nabla^{k} f_{0}(t)\right|^{p}+\left|\nabla^{k} f_{1}(t)\right|^{p}\right) d V(z) \frac{d t}{t}+\text { c.s. } \\
& \lesssim \int_{0}^{1} t^{-\theta k p}\left(\int_{\Omega_{t}} \delta^{k p}\left|\nabla^{k} f_{0}(t)\right|^{p} d V(z)+t^{k p} \int_{\Omega_{t}}\left|\nabla^{k} f_{1}(t)\right|^{p} d V(z)\right) \frac{d t}{t}+\text { c.s. } \\
& \lesssim \int_{0}^{1} t^{-\theta k p}\left(\left(\left\|f_{0}(t)\right\|_{0, p}\right)^{p}+\left(t^{k}\left\|f_{1}(t)\right\|_{k, p}\right)^{p}\right) \frac{d t}{t}+\text { c.s. } \\
& \lesssim \int_{0}^{1} t^{-\theta k p}\left(K\left(t^{k}, f\right)\right)^{p} \frac{d t}{t}+\text { c.s. } \\
& =1 / k \int_{0}^{1} t^{-\theta p}(K(t, f))^{p} \frac{d t}{t}+\text { c.s. } \\
& \lesssim\left(\|f\|_{\left.A^{0, p}, A^{k, p}\right)_{\theta, p}}\right)^{p} \text {. }
\end{aligned}
$$


Since $C^{\infty}(\bar{\Omega}) \cap O(\Omega)$ is dense in $A^{k, p}$, and hence in $\left(A^{0, p}, A^{k, p}\right)_{\theta, p}$, the above estimate implies that $\left(A^{0, p}, A^{k, p}\right)_{\theta, p}$ imbeds continuously into $A^{k \theta, p}$ (use that point evaluations are continuous in all the norms involved).

Before proceeding to prove the reverse imbedding, we would like to point out the following. For $1 \leq p$, the spaces under consideration are Banach spaces, and $A^{k, p}=\mathscr{D}\left(X^{k}\right)$, the domain of $X^{k}$, by Proposition 4 and Lemma 6 (recall that $\left.X f=\sum_{j=1}^{n} z_{j} \partial f / \partial z_{j}\right)$. Also recall the semigroup $\tau_{t}$, with $\tau_{t} f(z):=f\left(e^{-t} z\right)$. Then $-X$ is the generator of this semigroup. So we are interpolating between the Banach space $A^{0, p}$ and the domain of the $k$ th power of the generator of the semigroup $\tau_{t}$. This situation is well understood, and there are explicit formulas (in terms of $X^{k}$ and the semigroup) for the norms of the intermediate spaces (e.g., see [Bu-Be, $\S 3.5]$ or $[\mathrm{T}, \S 1.14 .5])$. Note that the Lipschitz condition on $b \Omega$ implies that the semigroup $\tau_{t}$ is analytic: $\tau_{\zeta} f(z):=f\left(e^{-\zeta} z\right)$ gives an analytic extension into an angular region. From these explicit formulas, it is not hard to see (by arguments analogous to the ones above) that the norm on the intermediate space is equivalent to \|\|$_{\theta k, p}$. The case $0<p<1$ is not covered by this, however. Again by using the radial maximal function (so as to be able to use a "monotone" version of a Hardy-inequality), one can treat this case as well.

Thus, let $f \in O(\Omega) \cap C^{\infty}(\bar{\Omega})$. For $t>0$, we must find decompositions $f=f_{0}(t)+f_{1}(t), f_{0}(t) \in A^{0, p}, f_{1}(t) \in A^{k, p}$, such that

$$
\int_{0}^{\infty} t^{-\theta p}\left(\left\|f_{0}(t)\right\|_{0, p}+t\left\|f_{1}(t)\right\|_{k, p}\right)^{p} \frac{d t}{t} \lesssim\|f\|_{\theta k, p}^{p} .
$$

First note that the integral in (5) equals

$$
k \int_{0}^{\infty} t^{-\theta k p}\left(\left\|f_{0}\left(t^{k}\right)\right\|_{0, p}+t^{k}\left\|f_{1}\left(t^{k}\right)\right\|_{k, p}\right)^{p} \frac{d t}{t} .
$$

We will estimate (6); this keeps the notation somewhat simpler. Again,

$$
\tau_{t} f(z):=f\left(e^{-t} z\right) .
$$

We set

$$
f_{0}\left(t^{k}\right)(z):=\frac{(-1)^{k} k}{t^{k}} \int_{0}^{t} s^{k-1}\left(\tau_{s}-I\right)^{k} f(z) d s
$$

and

$$
f_{1}\left(t^{k}\right)(z)=f(z)-f_{0}\left(t^{k}\right)(z)=k \sum_{j=1}^{k}(-1)^{j+1}\left(\begin{array}{l}
k \\
j
\end{array}\right) \frac{1}{t^{k}} \int_{0}^{t} s^{k-1}\left(\tau_{s}\right)^{j} f(z) d s .
$$

Formulas (8) and (9) are closely related to well-known formulas in the theory of interpolation of semigroups $(1 \leq p)$; compare [Bu-Be, p. 193]. 
We now estimate the contribution to (6) coming from $\left\|f_{0}\left(t^{k}\right)\right\|_{0, p}^{p}$. We have (10)

$$
\begin{aligned}
& \int_{0}^{\infty} t^{-\theta k p} \int_{\Omega}\left|f_{0}\left(t^{k}\right)(z)\right|^{p} d V(z) \frac{d t}{t} \\
& \lesssim \int_{0}^{\infty} t^{-\theta k p} \int_{\Omega}\left|\frac{1}{t^{k}} \int_{0}^{t} s^{k-1}\left(\tau_{s}-I\right)^{k} f(z) d s\right|^{p} d V(z) \frac{d t}{t}
\end{aligned}
$$

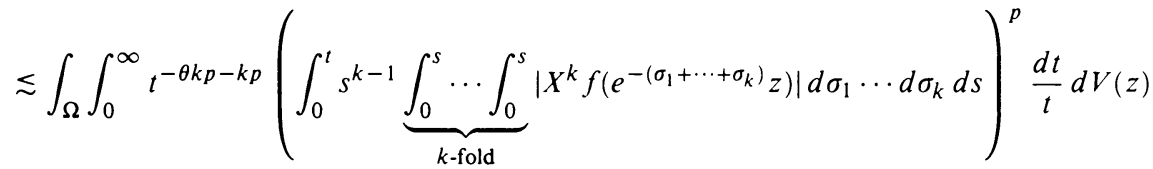

$$
\begin{aligned}
& \lesssim \int_{\Omega} \int_{0}^{\infty} t^{-\theta k p-k p}\left(t \cdot t^{k-1} \int_{0}^{t} \cdots \int_{0}^{t}\left|X^{k} f\left(e^{-\left(\sigma_{1}+\cdots+\sigma_{k}\right)} z\right)\right| d \sigma_{1} \cdots d \sigma_{k}\right)^{p} \frac{d t}{t} d V(z) \\
& \text { (11) } \lesssim \int_{\Omega} \int_{0}^{\infty} t^{-\theta k p}\left(\int_{0}^{k t} s^{k-1}\left|X^{k}\left(e^{-s} z\right)\right|^{*} d s\right)^{p} \frac{d t}{t} d V(z) \text {. }
\end{aligned}
$$

Here, the ${ }^{*}$ denotes again the radial maximal function when $0<p<1$ and the null symbol when $1 \leq p$ (i.e., $\left.|g(z)|^{*}=\sup _{t>0}\left|g\left(e^{-t} z\right)\right|\right)$. Applying the first inequality from Lemma 1 of $\S 5$ to the integration in $t$ (also changing variables $k t \rightarrow t)$, the last term above is dominated by

$$
\begin{aligned}
\int_{\Omega} \int_{0}^{\infty} t^{-\theta k p} t^{p} t^{k p-p}\left(\left|X^{k} f\left(e^{-t} z\right)\right|^{*}\right)^{p} \frac{d t}{t} d V(z) \\
\quad=\int_{0}^{\infty} t^{k p-\theta k p} \int_{\Omega}\left(\left|X^{k} f\left(e^{-t} z\right)\right|^{*}\right)^{p} d V(z) \frac{d t}{t} \\
\quad \lesssim \int_{0}^{1} t^{k p-\theta k p} \int_{\Omega_{t}}\left(\left|X^{k} f(z)\right|^{*}\right)^{p} d V(z) \frac{d t}{t}+\text { c.s. }
\end{aligned}
$$

Again, c.s. denotes compactly supported terms. Then, by an integration by parts trick similar to that in (4)

$$
\begin{aligned}
\int_{0}^{1} & \lesssim \int_{\Omega}\left(\delta^{k-\theta k}\left|X^{k} f\right|^{*}\right)^{p} d V(z)+\text { c.s. } \\
& \lesssim \int_{\Omega}\left(\delta^{k-\theta k}\left|X^{k} f\right|\right)^{p} d V(z)+\text { c.s. } \lesssim\left(\|f\|_{\theta k, p}\right)^{p}
\end{aligned}
$$

by Lemma 3 (note that $X^{k} f$ is analytic).

Next, we estimate the contribution in (6) coming from $f_{1}(t)$. It turns out that the contribution of each term in (9) is actually bounded by $\left(\|f\|_{\theta k, p}\right)^{p}$. 
The contribution from the $j$ th term is dominated (by Proposition 4)

$$
\begin{aligned}
\int_{0}^{\infty} & t^{-\theta k p} \int_{\Omega}\left(\int_{0}^{t} s^{k-1}\left|\left(\tau_{s}\right)^{j} X^{k} f(z)\right| d s\right)^{p} d V(z) \frac{d t}{t} \\
& \leq \int_{\Omega} \int_{0}^{\infty} t^{-\theta k p}\left(\int_{0}^{t} s^{k-1}\left|X^{k} f\left(e^{-j s} z\right)\right|^{*} d s\right)^{p} \frac{d t}{t} d V(z) \\
& \lesssim \int_{\Omega} \int_{0}^{\infty} t^{-\theta k p} t^{p} t^{k p-p}\left(\left|X^{k} f\left(e^{-j t} z\right)\right|^{*}\right)^{p} \frac{d t}{t} d V(z) \quad \text { Lemma 1, §5) } \\
& =\int_{0}^{\infty} t^{-\theta k p+k p} \int_{\Omega}\left(\left|X^{k} f\left(e^{-j t} z\right)\right|^{*}\right)^{p} d V(z) \frac{d t}{t} \\
& \lesssim \int_{\Omega}\left(\delta^{k-\theta k}\left|X^{k} f(z)\right|^{*}\right)^{p} d V(z)+\text { c.s. } \\
& \lesssim \int_{\Omega}\left(\delta^{k-\theta k}\left|X^{k} f(z)\right|\right)^{p} d V(z)+\text { c.s. } \lesssim\left(\|f\|_{\theta k, p}\right)^{p}
\end{aligned}
$$

with by now familiar arguments. We have thus shown that (6) is dominated by $\left(\|f\|_{\theta k, p}\right)^{p}$. This completes the proof of Theorem 1 (by the same density argument as in the first part).

It remains to finish the proof of Theorem 2. Again, this reduces to proving that

$$
\left(A^{0, \infty}, A^{k, \infty}\right)_{\theta, \infty}=A^{\theta k, \infty} .
$$

This proof is analogous to the previous one, so we omit the details (the density argument is not needed here, since the norms involved are sup-norms, and so integration by parts is not required).

\section{APPENDIX: HARDY'S INEQUALITIES FOR $0<p<1$}

The following pair of inequalities, usually referred to as Hardy's inequalities, is well known (see, e.g., [Ste, Appendix A.4]):

$$
\begin{aligned}
& \text { (1) }\left(\int_{0}^{\infty}\left(\int_{0}^{x} f(y) d y\right)^{p} x^{-r-1} d x\right)^{1 / p} \leq \frac{p}{r}\left(\int_{0}^{\infty}(y f(y))^{p} y^{-r-1} d y\right)^{1 / p}, \\
& \text { (2) }\left(\int_{0}^{\infty}\left(\int_{x}^{\infty} f(y) d y\right)^{p} x^{r-1} d x\right)^{1 / p} \leq \frac{p}{r}\left(\int_{0}^{\infty}(y f(y))^{p} y^{r-1} d y\right)^{1 / p},
\end{aligned}
$$

where $f \geq 0, p \geq 1$, and $r>0$.

(1) and (2) fail for $0<p<1$ in general. However, if attention is restricted to functions of the form $f(y)=y^{q} h(y)$, with $h(y)$ nonincreasing, (1) and (2) remain true. We mention that inequalities of this type are implicit in [B]. Also, a special case occurs in [B-L, p. 10] with the same idea of proof, namely discretization of the integrals. 
Lemma 1. Let $q \in \mathbf{R}, h(y) \geq 0$ and nonincreasing, $r>0$, and $0<p<1$. Then

$$
\begin{aligned}
& \left(\int_{0}^{\infty}\left(\int_{0}^{x} y^{q} h(y) d y\right)^{p} x^{-r-1} d x\right)^{1 / p} \\
& \quad \leq C(p, q, r)\left(\int_{0}^{\infty}\left(y^{q+1} h(y)\right)^{p} y^{-r-1} d y\right)^{1 / p}
\end{aligned}
$$

and

$$
\begin{aligned}
& \left(\int_{0}^{\infty}\left(\int_{x}^{\infty} y^{q} h(y) d y\right)^{p} x^{r-1} d x\right)^{1 / p} \\
& \quad \leq C(p, q, r)\left(\int_{0}^{\infty}\left(y^{q+1} h(y)\right)^{p} y^{r-1} d y\right)^{1 / p} .
\end{aligned}
$$

Remark 1. It is part of the lemma that finiteness of the right-hand side implies finiteness of the left-hand side.

Proof. We give the proof of (3); (4) is proved similarly; $C(p, q, r)$ may as usual vary from occurrence to occurrence.

It suffices to consider the case $q \geq 0$ (if $q<0, y^{q} h(y)$ is decreasing, so apply the $q=0$ case). Discretizing the integrals, using the monotonicity of the integrands involved and the fact that $0<p<1$, gives

$$
\begin{aligned}
\int_{0}^{\infty}\left(\int_{0}^{x} y^{q} h(y) d y\right)^{p} x^{-r-1} d x & \leq \sum_{k=-\infty}^{+\infty}\left(\sum_{j=-\infty}^{k} h\left(2^{j}\right) \int_{2^{\prime}}^{2^{j+1}} y^{q} d y\right)^{p} 2^{-r k} \\
& \leq C(p, q, r) \sum_{j=-\infty}^{+\infty}\left(h\left(2^{j}\right)\right)^{p} 2^{j p(q+1)} \sum_{k=j}^{\infty} 2^{-r k} \\
& \leq C(p, q, r) \sum_{j=-\infty}^{+\infty}\left(h\left(2^{j}\right)\right)^{p} 2^{j p(q+1)-j r} .
\end{aligned}
$$

Now

$$
2^{j p(q+1)-j r}=C(p, q, r) \int_{2^{j-1}}^{2^{\prime}} y^{(q+1) p-r-1} d y
$$

(also for $(q+1) p-r=0)$. (6) shows that the last term in (5) is dominated by the $p$ th power of the right-hand side of (3) (since $h$ is nonincreasing). The lemma is proved.

\section{REFERENCES}

[Ba] D. Barrett, Regularity of the Bergman projection and local geometry of domains, Duke Math. J. 53 (1986), 333-343.

[B] R. P. Boas, Jr., Inequalities for monotonic series, J. Math. Anal. Appl. 1 (1960), 121-126.

[Be] F. Beatrous, Estimates for derivatives of holomorphic functions in pseudoconvex domains, Math. Z. 191 (1986), 91-116. 
[B-L] J. Bergh and J. Löfström, Interpolation spaces, Grundlehren Math. Wiss., vol. 223, Springer, Berlin, 1976.

[Bu-Be] P. Butzer and H. Berens, Semi-groups of operators and approximation, Grundlehren Math. Wiss., vol. 145, Springer, Berlin, 1967.

[C-W] R. Coifman and G. Weiss, Extensions of Hardy spaces and their use in analysis, Bull. Amer. Math. Soc. 83 (1977), 569-645.

[D] J. Detraz, Classes de Bergman de fonctions harmoniques, Bull. Soc. Math. France 109 (1981), 259-268.

[Da] B. Dahlberg, On the Poisson integral for Lipschitz and $C^{1}$-domains, Studia Math. 66 (1979), 13-24.

[Du] P. Duren, Theory of $H^{p}$ spaces, Academic Press, New York, 1970.

[F-S] Ch. Fefferman and E. Stein, $H^{p}$ spaces of several variables, Acta Math. 129 (1972), 137-193.

[G] J. Garnett, Bounded analytic functions, Academic Press, New York, 1981.

[H] P. Henrici, Applied and computational complex analysis, vol. 2, Wiley, New York, 1977.

[H-Ph] E. Hille and R. Phillips, Functional analysis and semi-groups, Amer. Math. Soc. Colloq. Publ., vol. 31, Amer. Math. Soc., Providence, R. I., 1957.

[K1] S. Krantz, Intrinsic Lipschitz classes on manifolds with applications to complex function theory and estimates for the $\bar{\partial}$ and $\bar{\partial}_{b}$ equations, Manuscripta Math. 24 (1978), 351-378.

[K2] _ Function theory of several complex variables, Wiley, New York, 1982.

[K-P-S] S. Krein, J. Petunin, and E. Semenov, Interpolation of linear operators, Transl. Math. Monographs, vol. 54, Amer. Math. Soc., Providence, R. I., 1982.

[Li1] E. Ligocka, Estimates in Sobolev norms \|\|$_{p}^{s}$ for harmonic and holomorphic functions and interpolation between Sobolev and Hölder spaces of harmonic functions, Studia Math. 86 (1987), 225-271.

[Li2] __ On the space of Bloch harmonic functions and interpolation of spaces of harmonic and holomorphic functions, Studia Math. 87 (1987), 223-238.

[M] V. Maz'ja, Sobolev spaces, Springer, Berlin, 1985.

[Ste] E. Stein, Singular integrals and differentiability properties of functions, Princeton Univ. Press, 1970.

[T] H. Triebel, Interpolation theory, function spaces, differential operators, North-Holland, Amsterdam, 1978.

Department of Mathematics, Texas A \& M University, College Station, Texas 77843 